Gynäkologe 2015 • 48:414-414

DOI 10.1007/s00129-015-3721-0

Online publiziert: 10. Juni 2015

๑) Springer-Verlag Berlin Heidelberg 2015

\author{
G. Emons ${ }^{1} \cdot$ N. Maass $^{2} \cdot$ W. Jonat ${ }^{2}$ \\ 'Universitäts-Frauenklinik, Göttingen, Deutschland \\ ${ }^{2}$ Campus Kiel, Klinik für Gynäkologie und Geburtshilfe, Universitätsklinikum Schleswig-Holstein, Kiel, \\ Deutschland
}

\title{
Update gynäkologische Onkologie
}

Für das in den Industrieländern im-

Liebe Leserin, lieber Leser,

in der gynäkologischen Onkologie sind in den letzten Jahren in einem MaBe neue Erkenntnisse gewonnen worden, dass unser „Update“ von 2011 (Der Gynäkologe 44) dringend einer Aktualisierung bedarf.

Unser Verständnis der Entstehungsmechanismen des Ovarialkarzinoms befindet sich in einem grundlegenden Wandel. Die Entwicklung aus Inklusionszysten des ovariellen Oberflächenepithels ist nur ein Mechanismus neben der Genese aus Zellen der Tube und aus Endometriose. Mit hoher Wahrscheinlichkeit hat die unterschiedliche Genese der verschiedenen Ovarialkarzinomtypen auch erhebliche Auswirkungen auf die Wirksamkeit der eingesetzten Medikamente.

\section{》) Der fundamentale Wandel im Verständnis der Ätiopathogenese wird wohl auch Therapieoptionen verändern}

Das in den Industrienationen häufigste Malignom der primären weiblichen Genitalorgane, das Endometriumkarzinom, hat eine recht gute Prognose, wenn die typischen Frühsymptome ernst genommen werden. Bei Endometriumkarzinomen mit hohem Rezidivrisiko tritt zunehmend die Chemotherapie gegenüber der Strahlentherapie als wirksamere adjuvante Behandlungsstrategie in den Vordergrund. mer seltener auftretende Zervixkarzinom liegt inzwischen eine S3-Leitlinie vor. Mangels belastbarer Studiendaten basieren viele der Empfehlungen jedoch auf einem Expertenkonsensus. Neue, weniger invasive Operationstechniken bedürfen oft noch der Evaluation durch entsprechende Studien.

\section{》) Zunehmend setzen sich rekonstruktive, organerhaltende gegenüber "ultra"-radikalen Techniken durch}

Die Inzidenz des Vulvakarzinoms nimmt in Deutschland insbesondere bei jüngeren Frauen zu. Ätiologisch spielt neben der infektiösen Genese (humane Papillomaviren) die Entstehung aus einem Lichen sclerosus zunehmend eine Rolle. In der Therapie setzen sich anstelle der früher üblichen „ultra“-radikalen Verfahren organerhaltende und rekonstruktive Techniken durch, deren onkologische Sicherheit zum Teil schon belegt, zum Teil noch zu prüfen ist.

Dieses „Update“ wird mit Sicherheit nicht das letzte sein, es bietet aber eine State-of-the-Art-2015-Darstellung, verfasst von Experten zum jeweiligen Thema. Die Artikel sind bewusst kurz und prägnant gehalten. Für weitere Details ist die relevante Literatur angegeben.
Viel Spaß beim Lesen wünschen

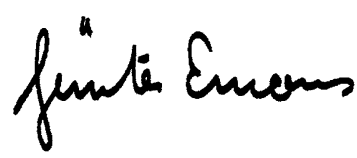

Prof. Dr. Günter Emons

N.T20ar

Prof. Dr. Nicolai Maass

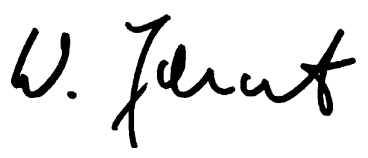

Prof. Dr. Walter Jonat

\section{Korrespondenzadresse}

Prof. Dr. G. Emons

Universitäts-Frauenklinik

Robert-Koch-Str. 40, 37075 Göttingen,

Deutschland

emons@med.uni-goettingen.de 